DOI: 10.17516/1997-1397-2021-14-6-815-821

УДК 517.9

\title{
Construction of Nonsingular Stress Fields for Non-Euclidean Model in Planar Deformations
}

\author{
Mikhail A. Guzev* \\ Perm National Research Polytechnic University \\ Perm, Russian Federation \\ Institute for Applied Mathematics \\ Far Eastern Branch of the Russian Academy of Sciences \\ Vladivostok, Russian Federation \\ Evgenii P. Riabokon ${ }^{\dagger}$ \\ Perm National Research Polytechnic University \\ Perm, Russian Federation
}

Received 10.04.2021, received in revised form 10.06.2021, accepted 20.08.2021

\begin{abstract}
A material with a microstructure is considered. A material is described on the basis of a non-Euclidean model of a continuous medium. In equilibrium, the total stress field is represented as the sum of elastic and self-balanced stresses, the parameterization of which is given through the scalar curvature of the Ricci tensor. It is proposed to use the spectral biharmonic equation to calculate the scalar curvature. Using the example of a plane strain state of a material, it is shown that the amplitude coefficients of elastic and self-balanced fields can be chosen so that singularities of the same type compensate each other in the full stress field.
\end{abstract}

Keywords: nonsingular stress field, planar deformation, microstructure, spectral biharmonic equation.

Citation: M. A. Guzev, E. P. Riabokon, Construction of Nonsingular Stress Fields for Non-Euclidean Model in Planar Deformations, J. Sib. Fed. Univ. Math. Phys., 2021, 14(6), 815-821.

DOI: 10.17516/1997-1397-2021-14-6-815-821.

\section{Introduction}

The singular behavior of the stress field in models of a continuous medium cannot be attributed to physical phenomena of the real world. Nevertheless, these singularities have been analyzed by researchers for a long time. The separation of their interests is associated, first of all, with the need to solve problems of different quality level, in which, in the general case, the use of various mathematical models is required to describe the internal properties of real materials during deformation. In particular, when studying the elastic-plastic behavior of materials, the necessity of introducing singular lines for analyzing the stress field around dislocations was considered by Burgers [1], while the word "singular" was used by him 83 times in that work. Approaches to interpreting the features of such fields in a physically meaningful way were proposed long ago [2].

Since the 1960s, the focus of theoretical research has been on the problem of constructing models for describing the microcharacteristics of materials in order to exclude a singularity and obtain a physically realistic result. A review of various approaches to overcoming singularities in the continuum theory of dislocations since the 1960s can be found in [3]. In that case, the authors proposed a nonsingular theory of dislocations, in which it is assumed that the core of

\footnotetext{
*guzev@iam.dvo.ru https://orcid.org/0000-0001-9344-154X

†riabokon.evgenii@gmail.com https://orcid.org/0000-0003-0555-3977

(C) Siberian Federal University. All rights reserved
} 
a dislocation is distributed in space. In [4], on the basis of the translational gauge theory of defects, an analytical representation was obtained for the fields of displacement, strain and stress that do not contain singularities in the dislocation core. The research cycle presented in [5-10] is devoted to the development of a nonsingular model for describing the field of elastic stresses and strains for dislocations and disclinations in the framework of a Mindlin's gradient theory. It should be noted that research groups motivated by Aifantis [11-14], also built nonsingular solutions of gradient elasticity for dislocations and cracks.

In continuum mechanics, each type of defect is associated with the state of internal stress of the continuum, for the description of which researchers in the 1950s suggested using nonEuclidean geometric objects $[15,16]$. Analysis of this problem for various decades can be found in [17-22]. The common approach of all authors is to use geometric non-Euclidean objects as variables characterizing the geometric structure of the incompatibility of the medium material elements. Although theoretical approaches in this direction are actively developing, the use of non-Euclidean objects for constructing a nonsingular model of defects has not received a wide representation in the scientific literature. Therefore, the purpose of this work is to fill this research gap.

\section{Internal stress field}

Internal stresses that exist in materials and do not disappear when external loads are removed are usually called self-balanced or residual. By definition [23, 24], the stress field inside the body has the property of self-balance if the total force and moment acting on the body are equal to zero. It was shown in [25] that the solutions $\sigma_{i j}$ equilibrium equations in the absence of mass forces inside the volume $V$ of a solid

$$
\frac{\partial \sigma_{i j}}{\partial x_{j}}=0
$$

have the following structure: $\sigma_{i j}=\tau_{i j}+T_{i j}$. Components $\sigma_{i j}$ include the field of elastic stresses $\tau_{i j}$ and the field of self-balanced stresses $T_{i j}$. In this case, the conditions for the absence of external forces on the surface of a solid are satisfied pointwise:

$$
\left.\left(\tau_{i j} n_{j}+T_{i j} n_{j}\right)\right|_{\partial V}=0,
$$

i.e. the combined action of these stresses allows it to maintain a given shape.

If, in the model of a continuous medium with an internal structure, non-Euclidean geometric objects are introduced to describe the internal stress state [26], then they allow to represent the stress field $T_{i j}$ in the following way:

$$
T_{i j}=\sigma_{0} l^{4}\left(\delta_{i j} \Delta R-\frac{\partial^{2} R}{\partial x^{i} \partial x^{j}}\right),
$$

where $R$ is a scalar curvature: trace of the Ricci tensor [27]; constants $\sigma_{0}$ and $l$ have the dimension of stress and length. The function $R$ is generally unknown. This geometric object was used to analyze the phenomenon of zonal disintegration [28]. Under the assumption that the internal energy of the medium depends on the thermodynamic variables quadratically, a stationary equation was obtained for $R$ in [26, 28]:

$$
\Delta^{2} R=\gamma R, \gamma \neq 0
$$

Parameter $\gamma$ depends on the spatial scale of the problem and has the dimension $\gamma=$ $=1 /[\text { dimension of length }]^{4}$. 


\section{Non-singular solutions}

To further concretize the above relations, consider the plane strain state of the material, assuming that the elastic stress field is equal to zero: $\tau_{i j}=0$. Obviously, the components $T_{i j}$ (3) satisfy the system of equations (1). Since $\Delta^{2}-\gamma=(\Delta+\sqrt{\gamma})(\Delta-\sqrt{\gamma})$, the solution for $R$ is given by the formula $R=R_{+}+R_{-}$, where $R_{+}, R_{-}$meet the requirements

$$
(\Delta+\sqrt{\gamma}) R_{+}=0, \quad(\Delta-\sqrt{\gamma}) R_{-}=0 .
$$

In order to construct the functions $R_{+}$and $R_{-}$, consider the polar coordinate system $(r, \varphi)$ and assume that they do not depend on $\varphi$. Introducing the variable $s=\sqrt[4]{\gamma} r$, we write relations $(5)$ in the following form:

$$
\frac{\partial^{2} R_{+}}{\partial s^{2}}+\frac{1}{s} \frac{\partial R_{+}}{\partial s}+R_{+}=0, \quad \frac{\partial^{2} R_{-}}{\partial s^{2}}+\frac{1}{s} \frac{\partial R_{-}}{\partial s}-R_{-}=0 .
$$

The solutions of equations (6) are determined in terms of the cylindrical functions:

$$
R_{+}(s)=a J_{0}(s)+b N_{0}(s), \quad R_{-}(s)=c K_{0}(s)+d I_{0}(s),
$$

where $J_{0}(s)$ and $N_{0}(s)$ are Bessel and Neumann functions respectively; $K_{0}(s)$ is a MacDonald function; $I_{0}(s)$ is a modified Bessel function; $a, b, c, d$ are amplitude coefficients that have the dimention $1 /[\text { dimension of lengt }]^{2}$. The only limited solution for all values of the argument is $R=R_{+}(s)=a J_{0}(s)$. In this case, the nonzero components of the stress field are determined from the formulas:

$$
\left\{\begin{array}{l}
T_{r r}=\sigma_{0} l^{4} \frac{1}{r} \frac{\partial R_{+}(s)}{\partial r}=-\sigma_{0} l^{4} \frac{a}{s} \sqrt{\gamma} J_{1}(s) \\
T_{\varphi \varphi}=\sigma_{0} l^{4} \frac{\partial^{2} R_{+}(s)}{\partial r^{2}}=\sigma_{0} l^{4} \sqrt{\gamma}\left(-\frac{1}{s} \frac{\partial R_{+}(s)}{\partial s}-R_{+}(s)\right)=\sigma_{0} l^{4} \sqrt{\gamma}\left(\frac{a}{s} J_{1}(s)-a J_{0}(s)\right)
\end{array}\right.
$$

in which we used the relations for the Bessel functions and the first equation in (5). Functions $T_{r r}, T_{\varphi \varphi}$ do not contain singularities at $s \rightarrow 0$, insofar as $J_{1}(s)$ has a first-order root at zero.

Consider the constructed solution for a circle with a radius under the condition that there are no forces on the boundary, then the boundary condition (2) is reduced to the vanishing of the radial stress on the circle: $\left.T_{r r}\right|_{r=a}=0$. From this and (8) we obtain

$$
J_{1}(\sqrt[4]{\gamma} a)=0
$$

From (9) it follows that parameter $\gamma(4)$ cannot be arbitrary, but is determined by the roots $s_{1 k}$ of the Bessel function $J_{1}(s): \gamma_{k}=\left(s_{1 k} / a\right)^{4}, k \geqslant 1$.

Now we assume that the elastic stress field $\tau_{i j}$ is nonzero:

$$
\frac{d \tau_{r r}}{d s}+\frac{\tau_{r r}-\tau_{\varphi \varphi}}{s}=0, \quad \Delta\left(\tau_{r r}+\tau_{\varphi \varphi}\right)=0, \quad \Delta=\frac{1}{s} \frac{\partial}{\partial s} s \frac{\partial}{\partial s} .
$$

The solutions of equations (10) are given by the formula:

$$
\tau_{r r}=c_{2} \ln s+\frac{c_{1}}{s^{2}}+\frac{1}{2} c_{2}+c_{3}, \quad \tau_{\varphi \varphi}=c_{2} \ln s-\frac{c_{1}}{s^{2}}+\frac{3}{2} c_{2}+c_{3} .
$$

Self-balanced stress field $T_{i j}(3)$ equals to

$$
T_{i j}=\sigma_{0} l^{4}\left(\delta_{i j} \Delta\left(R_{+}+R_{-}\right)-\frac{\partial^{2}\left(R_{+}+R_{-}\right)}{\partial x^{i} \partial x^{j}}\right) .
$$


Substituting (7) here and using the relations between the cylindrical functions, we obtain

$$
\begin{gathered}
T_{r r}=\sigma_{0} l^{4} \sqrt{\gamma}\left(-a \frac{J_{1}(s)}{s}-b \frac{N_{1}(s)}{s}-c \frac{K_{1}(s)}{s}+d \frac{I_{1}(s)}{s}\right), \\
T_{\varphi \varphi}=\sigma_{0} l^{4} \sqrt{\gamma}\left[a \frac{J_{1}(s)}{s}+b \frac{N_{1}(s)}{s}+c \frac{K_{1}(s)}{s}-d \frac{I_{1}(s)}{s}-a J_{0}(s)-b N_{0}(s)+c K_{0}(s)+d I_{0}(s)\right] .
\end{gathered}
$$

Elastic stress field $\tau_{i j}(11)$ has singular behavior at $s \rightarrow 0$. On the right-hand side of (13) and (14), individual terms also contain a singularity in the vicinity of zero, and such a contribution is made by the functions $N_{0}(s), K_{0}(s), N_{1}(s), K_{1}(s)$, since at $s \rightarrow 0$ they have the following asymptotics:

$$
N_{0}(s) \sim \frac{2}{\pi} \ln s, \quad K_{0}(s) \sim-\ln s, \quad N_{1}(s) \sim \frac{s}{\pi} \ln s-\frac{2}{\pi s}, \quad K_{1}(s) \sim \frac{1}{s}+\frac{s}{2} \ln s .
$$

Terms (13) and (14) containing $J_{1}(s), I_{1}(s)$ do not have singular behavior, because $J_{1}(s) \sim$ $I_{1}(s) \sim s / 2$ at $s \rightarrow 0$. Requirement for the absence of singular contributions in the total stress field $\sigma_{i j}=\tau_{i j}+T_{i j}$ leads to the fact that the coefficients in (11), (13) and (14) cannot be arbitrary: they should be chosen so that singularities of the same type compensate each other. Leading on $s$ the singularity is of $1 / s^{2}$ order. Equating the combination of coefficients to zero at $1 / s^{2}$ in $\sigma_{r r}=\tau_{r r}+T_{r r}, \sigma_{\varphi \varphi}=\tau_{\varphi \varphi}+T_{\varphi \varphi}$, we obtain

$$
c_{1}=\sigma_{0} l^{4} \sqrt{\gamma}\left(-\frac{2 b}{\pi}+c\right) .
$$

Note that the requirement that there is no singularity for $\sigma_{r r}, \sigma_{\varphi \varphi}$ does not lead to the appearance of two independent conditions and has the form (16). The next singular contribution to (13) and (14) contains $\ln s$, then the requirement that the coefficients vanish at $\ln s$ gives

$$
c_{2}=\sigma_{0} l^{4} \sqrt{\gamma}\left(\frac{b}{\pi}+\frac{c}{2}\right)
$$

Thus, under conditions (16), (17), the stress fields $\sigma_{r r}, \sigma_{\varphi \varphi}$ do not contain singular contributions.

\section{Conclusion}

The presence of an internal microstructure in the material forced us to single out the contributions in the total stress field that characterize its distribution in the material. The use of a non-Euclidean model of a continuous medium allowed to parameterize the corresponding internal stresses in terms of the scalar curvature of the Ricci tensor. Using the obtained relations to describe the plane strain state of the material, we obtained an analytical representation for the total stress field. The condition of the absence of singular contributions in it led to a consistent choice of amplitude coefficients in the solution for the fields, providing compensation for singularities in the total stress field. The performed procedure allows to construct a nonsingular solution for a plane strain state of a continuous medium based on a non-Euclidean model.

The investigation was financially supported by the Russian Science Foundation (project 1919-00408). 


\section{References}

[1] J.M.Burgers, Physics. Some Considerations on the Fields of Stress Connected With Dislocations in a Regular Crystal Lattice, I, II, in: F.T.M. Nieuwstadt, J.A. Steketee (Eds.), Selected Papers of J. M. Burgers, Springer, Dordrecht, 1995, 335-389.

DOI: 10.1007/978-94-011-0195-0-11

[2] J.D.Eshelby, The Continuum Theory of Lattice Defects, Solid State Physics, 1956, 79-144. DOI: $10.1016 /$ S0081-1947(08)60132-0

[3] W.Cai, A.Arsenlis, C.R.Weinberger, V.V.Bulatov, A Non-Singular Continuum Theory of Dislocations, Journal of the Mechanics and Physics of Solids, 54(2006), no. 3, 561-587. DOI: $10.1016 /$ j.jmps.2005.09.005

[4] M.Lazar, A Nonsingular Solution of the Edge Dislocation in the Gauge Theory of Dislocations, Journal of Physics A: Mathematical and General, 36(2003), no. 5, 1415-1437. DOI: $10.1088 / 0305-4470 / 36 / 5 / 316$

[5] M.Lazar, G.A.Maugin, Nonsingular stress and strain fields of dislocations and disclinations in first strain gradient elasticity, International Journal of Engineering Science, 43(2005), no. 13-14, 1157-1184. DOI: 10.1016/j.ijengsci.2005.01.006

[6] M.Lazar, Non-Singular Dislocation Loops in Gradient Elasticity, Physics Letters A, 376(2012), no. 21, 1757-1758. DOI: 10.1016/j.physleta.2012.04.009

[7] M.Lazar, The Fundamentals of Non-Singular Dislocations in the Theory of Gradient Elasticity: Dislocation Loops and Straight Dislocations, International Journal of Solids and Structures, 50(2013), no. 2, 352-362. DOI: 10.1016/j.ijsolstr.2012.09.017

[8] M.Lazar, G.Po, The Non-Singular Green Tensor of Gradient Anisotropic Elasticity of Helmholtz Type, European Journal of Mechanics - A/Solids, 50(2015), 152-362.

DOI:10.1016/j.euromechsol.2014.10.006

[9] M.Lazar, G.Po, The Non-Singular Green Tensor of Gradient Anisotropic Elasticity of Helmholtz Type, Physics Letters A, 379(2015), no. 24-25, 1538-1543.

DOI: $10.1016 /$ j.physleta.2015.03.027

[10] G.Po, M.Lazar, N.C.Admal, N.Ghoniem, A Non-Singular Theory of Dislocations in Anisotropic Crystals, International Journal of Plasticity, 103(2018), 1-22.

DOI: $10.1016 /$ j.ijplas.2017.10.003

[11] J.Kioseoglou, I.Konstantopoulos, G.Ribarik, G.P.Dimitrakopulos, E.C.Aifantis, Nonsingular Dislocation and Crack Fields: Implications to Small Volumes, Microsystem Technologies, 15(2008), no. 1, 117-121. DOI: 10.1007/s00542-008-0700-6

[12] E.C.Aifantis, A note on Gradient Elasticity and Nonsingular Crack Fields, Journal of the Mechanical Behavior of Materials, 20(2012), no. 4-5, 103-105.

DOI: $10.1515 / \mathrm{jmbm}-2012-0002$

[13] I.Konstantopoulos, E.C.Aifantis, Gradient Elasticity Applied to a Crack, Journal of the Mechanical Behavior of Materials, 22(2013), no. 5-6, 193-201.

DOI: $10.1515 / \mathrm{jmbm}-2013-0026$

[14] K.Parisis, I.Konstantopoulos, E.C.Aifantis, Nonsingular Solutions of GradEla Models for Dislocations: An Extension to Fractional GradEla, Journal of Micromechanics and Molecular Physics, 3(2018), no. 3-4, 193-201. DOI: 10.1142/S2424913018400131 
[15] K.Kondo, On the Geometrical and Physical Foundations of the Theory of Yielding, Proc. Japan. Nat. Congr. Apll. Mech, 2, 1952, 41-47.

[16] B.A.Bilby, R.Bullough, E.Smith, Continuous Distributions of Dislocations: a New Application of the Methods of Non-Reimannian Geometry, Proc. Roy. Soc., A231, 1955, 263-267. DOI: $10.1098 /$ rspa.1955.0171

[17] R.Stojanovic, Equilibrium Conditions for Internal Stresses in Non-Euclidian Continua and Stress Space, International Journal of Engineering Science, 1(1963), no. 3, 323-327. DOI: 10.1016/0020-7225(63)90010-7

[18] S.Minagawa, On the Stress Functions in Elastodynamics, Acta Mechanica, 24(1976), no. 3, 209-217. DOI: 10.1007 /BF01190371

[19] E.Kroner, Incompatibility, Defects, and Stress Functions in the Mechanics of Generalized Continua, International Journal of Solids and Structures, 21(1985), no. 7, 747-756. DOI: 10.1016/0020-7683(85)90077-0

[20] D.G.B.Edelen, A Correct, Globally Defined Solution of the Screw Dislocation Problem in the Gauge Theory of Defects, International Journal of Engineering Science, 34(1996), no. 1, 81-86. DOI: 10.1016/0020-7225(95)00081-x

[21] M.A.Guzev, Non-Euclidean Models of Elastoplastic Materials with Structure Defects, Lambert Academic Publishing, 2010.

[22] A.Yavari, A.Goriely, Riemann-Cartan Geometry of Nonlinear Disclination Mechanics, International Journal of Engineering Science, 34(2012), no. 1, 81-86.

DOI: $10.1177 / 1081286511436137$

[23] M.E.Gurtin, A Generalization of the Beltrami Stress Functions in Continuum Mechanics, Archive for Rational Mechanics and Analysis, 13(1963), no. 1, 321-329.

DOI: $10.1007 /$ BSF01262700

[24] V.P.Myasnikov, M.A.Guzev, A.A.Ushakov, Self-Equilibrated Stress Fields in a Continuous Medium, Journal of Applied Mechanics and Technical Physics, 45(2004), no. 4, 558-564. DOI: 10.1023/B:JAMT.0000030334.32046.e6

[25] V.P.Myasnikov, M.A.Guzev, Geometric Model of Internal Self-Equilibrated Stresses in Solids, Doklady Physics, 46(2001), no. 10, 740-741. DOI: 10.1134/1.1415593

[26] M.A.Guzev, Structure of Kinematic and Force Fields in the Riemannian Continuum Model, Journal of Applied Mechanics and Technical Physics, 52(2003), no. 5, 709-716.

DOI: $10.1134 /$ S002189441105004X

[27] S.P.Novikov, I.A.Taimanov, Modern Geometric Structures and Fields. Graduate Studies in Mathematics, American Mathematical Society, 71(2006). DOI: 10.1090/gsm/071

[28] M.A.Guzev, A.A.Paroshin, Non-Euclidean Model of the Zonal Disintegration of Rocks Around an Underground Working, Journal of Applied Mechanics and Technical Physics, 42(2001), no. 1, 131-139. DOI: 10.1023/A:1018877015940 


\title{
Построение несингулярных полей напряжений в неевклидовой модели для плоско-деформированного состояния сплошной среды
}

Михаил А. Гузев

Пермский национальный исследовательский политехнический университет

Пермь, Российская Федерация

Институт прикладной математики Дальневосточного отделения Российской академии наук

Владивосток, Российская Федерация

Евгений П. Рябоконь

Пермский национальный исследовательский политехнический университет

Пермь, Российская Федерация

\begin{abstract}
Аннотация. Рассматривается материал с микроструктурой, описание которой выполняется на основе неевклидовой модели сплошной среды. В равновесии полное поле напряжений представлено в виде суммы упругих и самоуравновешенных напряжений, параметризация которых дается через скалярную кривизну тензора Риччи. Для вычисления скалярной кривизны предлагается использовать спектральное бигармоническое уравнение. На примере плоско-деформированного состояния материала показано, что амплитудные коэффициенты упругих и самоуравновешенных полей можно выбрать так, чтобы сингулярности одного типа компенсировали друг друга в полном поле напряжений.
\end{abstract}

Ключевые слова: несингулярное поле напряжений, плоская деформация, микроструктура, спектральное бигармоническое уравнение. 